\title{
Long-Term Outcome of Bladder Augmentation Using Living-Related Partial Bladder Transplantation in Rats
}

\author{
ATSUYUKI YAMATAKA, KUN WANG, YOSHIFUMI KATO, YASUHIRO OKADA, \\ HIROYUKI KOBAYASHI, GEOFFREY J. LANE, HIROYUKI KOGA, NORIYOSHI SUEYOSHI, AND \\ TAKESHI MIYANO \\ Department of Pediatric General and Urogenital Surgery [A.Y., K.W., Y.K., Y.O., H.Kobayashi, G.J.L., \\ H.Koga, T.M.], Division of Biomedical Imaging Research [N.S.], Biomedical Research Centre, Graduate \\ School of Medicine Juntendo University School of Medicine, Tokyo 113-8421, Japan
}

\begin{abstract}
Long-term histopathologic changes after bladder augmentation (BA) in rats using living-related partial bladder transplantation (LPBTx) or conventional ileocystoplasty (ICP) were compared. In this study, BA $(n=37)$, LPBTx $(n=18)$, and ICP $(n$ $=19$ ) were performed in 16-wk-old Lewis rats. Five donors and seven nontransplanted normal Lewis rats (controls) were also studied. Rats that survived $>10$ mo after BA were killed after blood biochemistry and neobladder imaging. Harvested bladders were examined with hematoxylin and eosin and proliferating cell nuclear antigen (PCNA). When the rats were killed, there were 16 rats in the LPBTx group and 12 rats in the ICP group; ICP rats were significantly smaller than LPBTx rats $(p<0.05)$. Mean duration of follow-up for the LPBTX group was $17.3 \mathrm{mo}$, for the ICP group was $13.7 \mathrm{mo}$, for the donor group was $16.1 \mathrm{mo}$, and for the control group was 19.7 mo. Mean serum $\mathrm{pH}$ in the LPBTx group was $7.41 \pm 0.78$ and in the ICP group was $7.25 \pm 0.38$. Mean base excess in the ICP group was significantly lower than in the LPBTx group $(p<0.05)$. Incidence of bladder calculi in
\end{abstract}

\section{ABSTRACT}

the LPBTx group (6.3\%) was significantly lower than in the ICP group $(33.3 \% ; p<0.05)$. There was no dysplasia/malignancy/ increase in PCNA in the LPBTx group. PCNA increased in the ICP group, compared with controls ( $p<0.05)$; two $(16.7 \%)$ of 12 of ICP rats had dysplasia with mitosis. Bladder capacity increased in LPBTx and ICP compared with controls (both $p<$ 0.05). We hope to show that BA using LPBTx may result in a neobladder with fewer complications than BA using ICP; LPBTx may also decrease the risk for malignancy. (Pediatr Res 57: 738-743, 2005)
BA, bladder augmentation
ICP, conventional ileocystoplasty
LPBTx, living-related partial bladder transplantation
PCNA, proliferating cell nuclear antigen
PCNA-LI, PCNA labeling index

\section{Abbreviations}

Segments of gastrointestinal tract are commonly used for bladder augmentation (BA) to treat patients with neurogenic bladder or bladder exstrophy $(1,2)$. However, when gastrointestinal tissue is in constant contact with urine, various significant post-BA complications, such as urolithiasis, metabolic acidosis, bladder perforation, increased mucous production, and tumor (1-3), can develop. In particular, any possible risk for malignancy in the intestinal segment used for BA is a matter of grave concern $(1,3)$. Filmer and Spencer (4) reviewed the development of cancer in augmented bladders in humans and estimated that the latency between BA and tumor development is long, with a mean of $18 \mathrm{y}$.

Received February 13, 2004; accepted September 22, 2004.

Correspondence: Atsuyuki Yamataka, M.D., Department of Pediatric General \& Urogenital Surgery, Juntendo University School of Medicine, 2-1-1 Hongo, Bunkyo-ku, Tokyo 113-8421, Japan; e-mail: yama@med.juntendo.ac.jp.

Supported by a Grant-in-Aid for Scientific Research (C2) (No. 15591893) from the Ministry of Education, Culture, Sports, Science, and Technology, Japan.

DOI: 10.1203/01.PDR.0000156513.42054.D7
To overcome post-BA complications associated with using enteric tissue, we developed a novel experimental technique for BA, which we tested in rats: living-related partial bladder transplantation (LPBTx) (5). The short-term results were very encouraging and have already been published. Here, we report the long-term results in a new series of Lewis rats, focusing on the risk for carcinogenesis in the neobladder. Outcome is compared with that for conventional ileocystoplasty (ICP).

\section{METHODS}

All experimental protocols were approved by the Institutional Animal Care and Use Committee at Juntendo University School of Medicine (Experimental Protocol No. 140-110) in accordance with guidelines set forth in the National Institutes of Health Guide for the Care and Use of Laboratory Animals.

Before surgery, rats were deprived of food for $1 \mathrm{~d}$ and given atropine $(0.04$ $\mathrm{mg} / \mathrm{kg}$ ) by s.c. injection and double antibiotic therapy with cefmetazole sodium $(100 \mathrm{mg} / \mathrm{kg})$ and amikacin sulfate $(6 \mathrm{mg} / \mathrm{kg})$ by i.m. injection on the day of surgery. The anesthetic agent used was ketamine $(15 \mathrm{mg} / \mathrm{kg})$ given by i.p. injection. 
An operating microscope with $\times 3.5$ magnification was used to perform BA. Altogether, 37 Lewis rats underwent BA using either LPBTx $(n=18)$ or ICP $(n=19)$.

LPBTx. For LPBTx, the cranial one third of the bladder from a 16-wk-old adult female Lewis rat that weighed 195-230 g (donor) was excised and transplanted into a pouch created in the distal omentum of one of its 6-wk-old female offspring that weighed $110-145 \mathrm{~g}$ (recipient). The bladder remnant of the donor rat was closed using single-layer interrupted sutures. No immunosuppressant was used in the recipient. Neovascularization of the transplanted bladder dome was allowed to progress for $2 \mathrm{wk}$, then a laparotomy was performed to excise the cranial quarter of the recipient's native bladder mobilize the bladder graft with its omental pedicle into the pelvis, and anastomose it to the recipient native bladder remnant. A single layer of interrupted, full-thickness, 7/0 polydioxanone synthetic absorbable sutures was used for the anastomosis, and omentum was used to reinforce the anastomotic line.

Ileocystoplasty. For ICP, 16-wk-old female rats that weighed 200-230 g were used so that the age of the ileal patch mucosa would match the age of the donor bladder mucosa used in the LPBTx group. The cranial quarter of the bladder was excised, and then a vascularized rectangular patch of ileum made by detubularizing an isolated segment of ileum and incising it along its antimesenteric border was anastomosed using full-thickness, interrupted, single-layer sutures. Bowel continuity was reestablished through an end-to-end anastomosis using interrupted inverting sutures. Sutures that were used for BA and bowel anastomosis all were $7 / 0$ polydioxanone synthetic absorbable sutures. The abdominal wall and skin were closed with $3 / 0$ polydioxanone sutures.

Postoperative follow-up. After surgery, all rats were given unrestricted access to standard food and water. They were examined daily. Animals seemed to void spontaneously throughout the study, and no bladder catheterization or diversion of urine was required.

Long-term follow-up. Rats that survived $>10$ mo were killed and used in this study. Rats that died unexpectedly within 10 mo of BA were refrigerated autopsied within $24 \mathrm{~h}$ of death, but were excluded because they were not considered to be long-term survivors. Just before they were killed, rats were weighed and had blood taken to measure acid/base balance and renal function (especially creatinine and blood urea nitrogen), and neobladder imaging was performed using 30\% iopromide under anesthesia. All neobladder were excised in toto after death or killing. Five donor rats (donor group) and seven nontransplanted, normal rats (control group) had the same investigations as the study group rats for at least $10 \mathrm{mo}$, then were killed.

Histopathology. Excised bladders were fixed in 10\% neutral-buffered formalin and examined blindly by a single pathologist (N.S.) using hematoxylin and eosin staining and proliferating cell nuclear antigen (PCNA) immunostaining to detect preneoplastic or neoplastic changes in the bladder mucosa such as dysplasia and carcinoma. The upper urinary tract was examined grossly for hydronephrosis or other physical abnormalities at the time of killing/autopsy.

A PCNA labeling index (PCNA-LI) $(6,7)$ was used to classify abnormal cell distribution. The number of PCNA-positive cells present in 500 epithelial cells was counted blindly in each specimen under $\times 200$ magnification by the same observer and then expressed as a percentage of the total number of cells evaluated. PCNA-LI calculated for five randomly selected sections from each bladder specimen were averaged to give the final PCNA-LI.

Statistical analysis. StatView software (SAS Institute, Cary, NC) was used for statistical analysis. Laboratory data, body weight, and bladder capacity for all rats were expressed as mean \pm SD. Differences in data between control, donor, LPBTx, and ICP groups were compared using the $t$ test. Differences in the incidences of bladder calculi and mucosal abnormalities in the augmented bladder between the LPBTx and ICP groups were compared using the Fisher exact probability test. Results that demonstrated $p<0.05$ were considered significant.

\section{RESULTS}

Survival. In general, ICP rats seemed weaker and less active than LPBTx rats. ICP rats were significantly smaller than controls $(p<0.05)$, but there were no significant differences between LPBTx rats and controls and between donors and controls. Mean weights at the time of killing in the control, donor, LPBTx, and ICP groups were $332.9 \pm 18.0,336.0 \pm$ 24.1, $320.6 \pm 37.7$, and $273.3 \pm 61.8 \mathrm{~g}$, respectively (Fig. 1).

Mean duration of follow-up was $17.3 \mathrm{mo}$ (range, 11-21 mo) in the LPBTx group, 13.7 mo (range, 10-16 mo) in the ICP

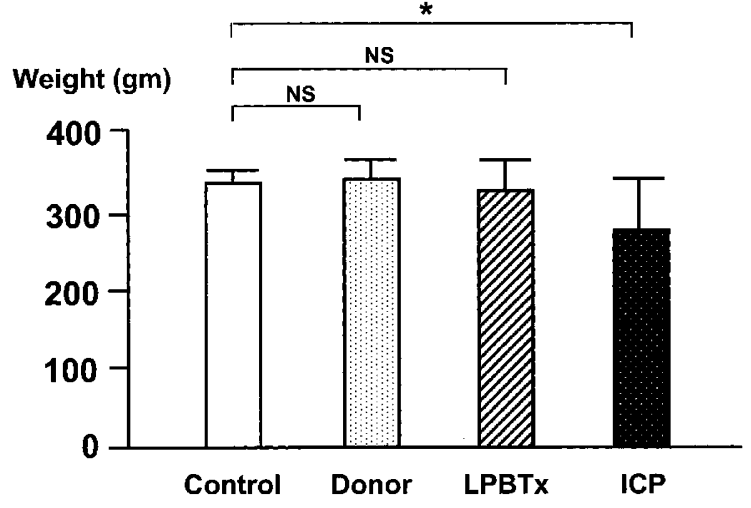

Figure 1. Mean weights at killing in the control $(n=7)$, donor $(n=5)$, LPBTx $(n=16)$, and ICP groups $(n=12)$. Rats in the ICP group were significantly smaller than controls $(p<0.05)$, but there were no significant differences between LPBTx rats and controls and between donors and controls. $* p<0.05$

group, and $16.1 \mathrm{mo}$ (range, 11-21 mo) in the donor group. Mean age of the control group at killing was 19.7 mo (range, $14-25 \mathrm{mo}$ ).

In the BA groups, two rats in the LPBTx group and four rats in the ICP group died within 3 mo of BA. Causes of death identified at autopsy were adhesive bowel obstruction (two in the LPBTx group and one in the ICP group), vesico-intestinal anastomotic breakdown (urine leakage) in two ICP rats, and unknown in one ICP rat. Three other rats died in the ICP group before being killed. Causes of death identified at autopsy were urinary tract infection secondary to grossly dilated upper urinary tract and bladder calculi in two and unknown in one. Unfortunately, these three rats were already in advanced stages of autolysis at the time of autopsy, precluding adequate histologic evaluation. There were no further deaths in the LPBTx group. As a result, 12 ICP rats and 16 LPBTx rats survived until killing, and nine rats died in the BA groups (two in the LPBTx group and seven in the ICP group) and were excluded. There were no deaths in the donor or control groups until killing at $10 \mathrm{mo}$.

Biochemistry. Creatinine was normal in all killed rats. Just before killing, mean serum $\mathrm{pH}$ in the LPBTx group was $7.41 \pm$ 0.78 and in the ICP group was $7.25 \pm 0.38$. Mean blood urea nitrogen in the LPBTx group was $17.2 \pm 1.7 \mathrm{mg} / \mathrm{dL}$ and in the ICP group was $29.4 \pm 6.1 \mathrm{mg} / \mathrm{dL}$, which was significantly higher $(p<0.05)$. Mean base excess in the LPBTx group was $0.89 \pm 0.76 \mathrm{mM}$. In contrast, mean base excess in the ICP group was significantly lower at $-4.50 \pm 2.67 \mathrm{mM}$ compared with the LPBTx group $(p<0.05)$.

Neobladder shape and size. Neobladder imaging in the BA groups showed bladders that were nicely augmented (Fig. 2). Bladder calculi were found in four $(33.3 \%)$ ICP rats and in one (6.3\%) LPBTx rat. There was no correlation between the incidence of bladder calculi and presence of mucosal abnormalities (inflammation, hyperplasia, metaplasia, or dysplasia) in augmented bladders.

Mean bladder capacities in the control, donor, LPBTx, and ICP groups were $1.3 \pm 0.3,1.2 \pm 0.4,2.1 \pm 0.9$, and $5.9 \pm 5.1$ $\mathrm{mL}$, respectively (Fig. 3). Bladder capacity was significantly 

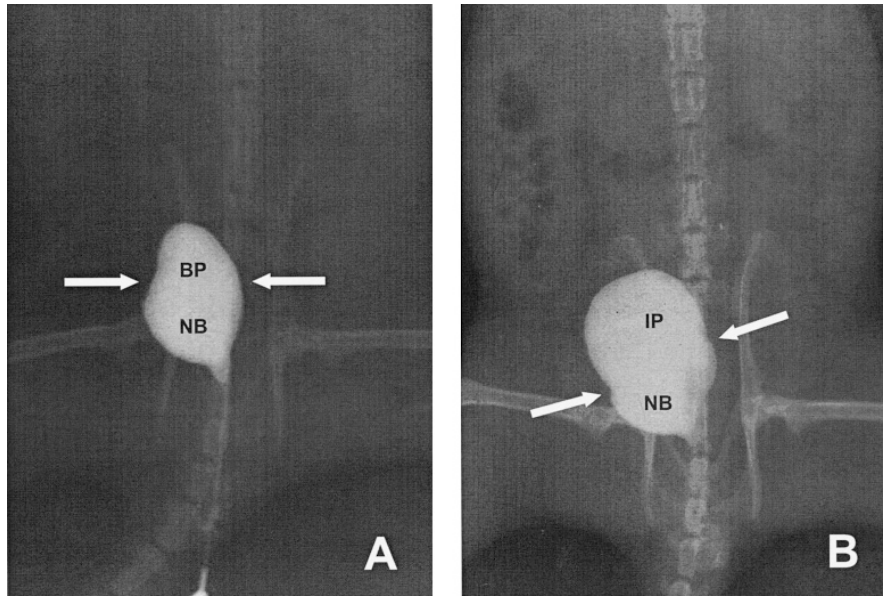

Figure 2. Neobladder imaging in the LPBTx $(A)$ and ICP $(B)$ groups showed bladders that were nicely augmented. Arrows indicate the vesicovesical anastomosis in the LPBTx group and the ileo-vesical anastomosis in the ICP group. $\mathrm{BP}$, bladder patch; NB, native bladder; IP, ileal patch.

\section{Bladder capacity $(\mathrm{mL})$}

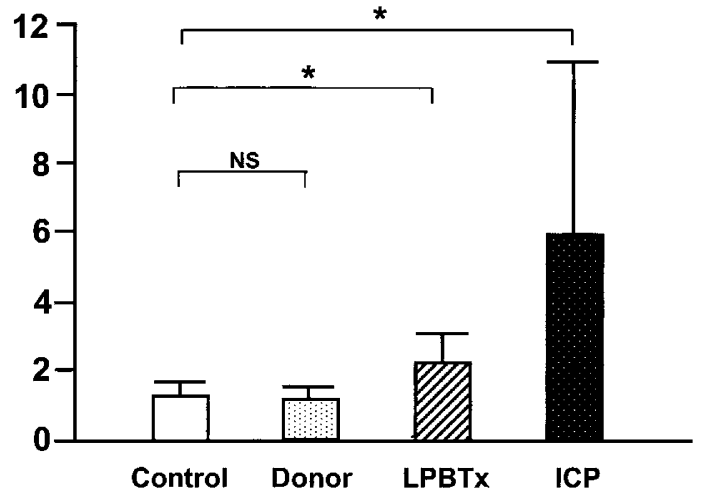

Figure 3. Bladder capacities in the control $(n=7)$, donor $(n=5)$, LPBTx $(n$ $=16)$, and ICP groups $(n=12)$. Mean bladder capacities were significantly increased in both the LPBTx and the ICP groups compared with controls $(p<$ 0.05 for both). There were no significant differences between donors and controls. $* p<0.05$.

increased in both the LPBTx and ICP groups compared with controls $(p<0.05$ for both). In LPBTx rats, mean bladder capacity was increased but less than double the mean control capacity. In contrast, in the ICP group, the mean bladder capacity was four times that of controls. In fact, three ICP rats had huge bladders with capacities of 17.6, 13.3, and $9.0 \mathrm{~mL}$, respectively. In two of the bladders complicated by calculi, the actual capacity was very small because the bladder lumen was full of multiple calculi (Fig. 4). Bladder dynamics were not assessed in further detail because there is no reliable way to measure voiding capacity and voiding pressure in rats at our institution. Once our technique is successful in larger animals, complete urodynamic studies will be possible, providing further information about the potential for this technique to be applied in humans.

Histopathology. Macroscopically, there was no dilation of the upper urinary tract seen in any LPBTx rat at killing, but in the ICP group, hydroureteronephrosis was present in three kidneys in three rats; only one of these rats also had bladder calculi.

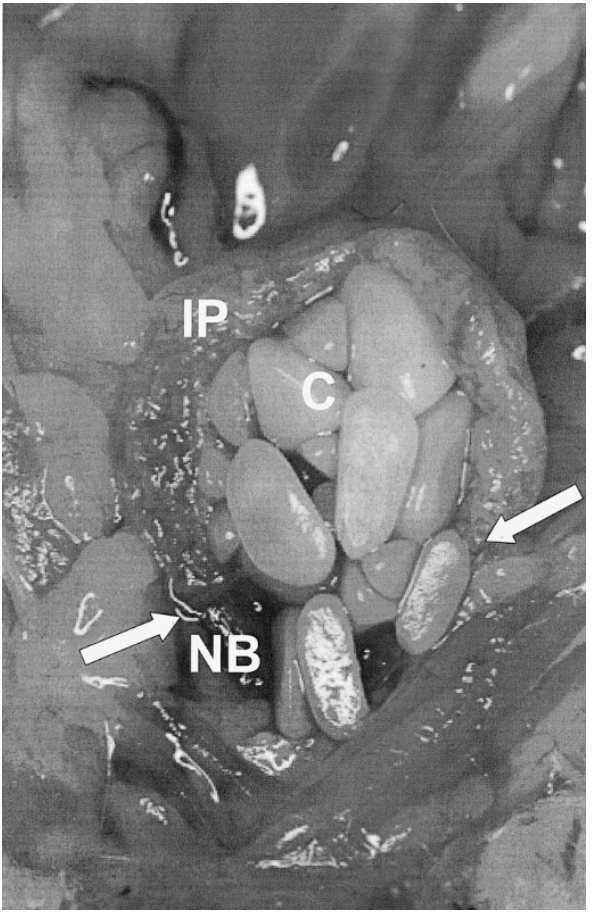

Figure 4. Bladder calculi in an ICP rat. The neobladder lumen is full of multiple calculi (C). Arrows indicate the ileovesical anastomosis between the IP and the NB.

Histologic findings are shown in Table 1. In the control and donor groups, the only abnormality identified was chronic inflammation. In both the native bladders and bladder patches in LPBTx rats, there was no evidence of metaplasia, mitosis, dysplasia, or malignancy (Fig. 5). Chronic inflammation and/or hyperplasia was identified generally but more intensely at or near the vesicovesical anastomosis. In all ICP rats, chronic inflammation, hyperplasia, and/or metaplasia was more generalized both in the native bladder and in the ileal patch mucosa (Fig. 6) but more intense at or near the vesico-intestinal anastomosis. Dysplasia with mitosis was identified in the ileal patch mucosa near its anastomosis with the native bladder in two (16.7\%) of 12 ICP rats (Fig. 7), whereas it was not identified in the remaining 10 .

In ICP rats, PCNA-LI was significantly increased in both the native bladder and the ileal patch mucosa near or at the vesico-intestinal anastomosis (Fig. 8), compared with controls (both $p<0.05$ ). In contrast, there was no increase in PCNA-LI for both bladder patches and native bladders in LPBTx rats.

\section{DISCUSSION}

Incorporation of intestinal segments into the urinary tract is associated with significant long-term complications (1-4). Therefore, surgeons have tried to avoid using enteric tissue for BA and have tried techniques such as autoaugmentation, ureterocystoplasty, tissue expansion, seromuscular grafts, matrices for tissue regeneration, and tissue engineering using cell transplantation (8).

Because BA is frequently performed in children, there is growing concern about the risk for carcinogenesis associated with this procedure over time. Rats have a life span of $\sim 2-3$ y 
Table 1. Histopathology of augmented bladders

\begin{tabular}{|c|c|c|c|c|c|c|}
\hline Specimens & Control & Donor & $\begin{array}{l}\text { LPBTx native } \\
\text { bladder }\end{array}$ & $\begin{array}{c}\text { LPBTx } \\
\text { bladder patch }\end{array}$ & $\begin{array}{l}\text { ICP native } \\
\text { bladder }\end{array}$ & $\begin{array}{l}\text { ICP ileal } \\
\text { patch }\end{array}$ \\
\hline Total no. & 7 & 5 & 16 & 16 & 12 & 12 \\
\hline No. of normal & 6 & 4 & 8 & 6 & 0 & 0 \\
\hline \multicolumn{7}{|l|}{ Abnormal findings } \\
\hline Chronic inflammation & 1 & 1 & 2 & 2 & 9 & 11 \\
\hline Hyperplasia & 0 & 0 & 8 & 10 & 5 & 9 \\
\hline Dysplasia & 0 & 0 & 0 & 0 & 0 & 2 \\
\hline Carcinoma & 0 & 0 & 0 & 0 & 0 & 0 \\
\hline
\end{tabular}

* Abnormal findings were more intense near or at the vesicovesical anastomosis in the LPBTx group and near or at the ileovesical anastomosis in the ICP group.

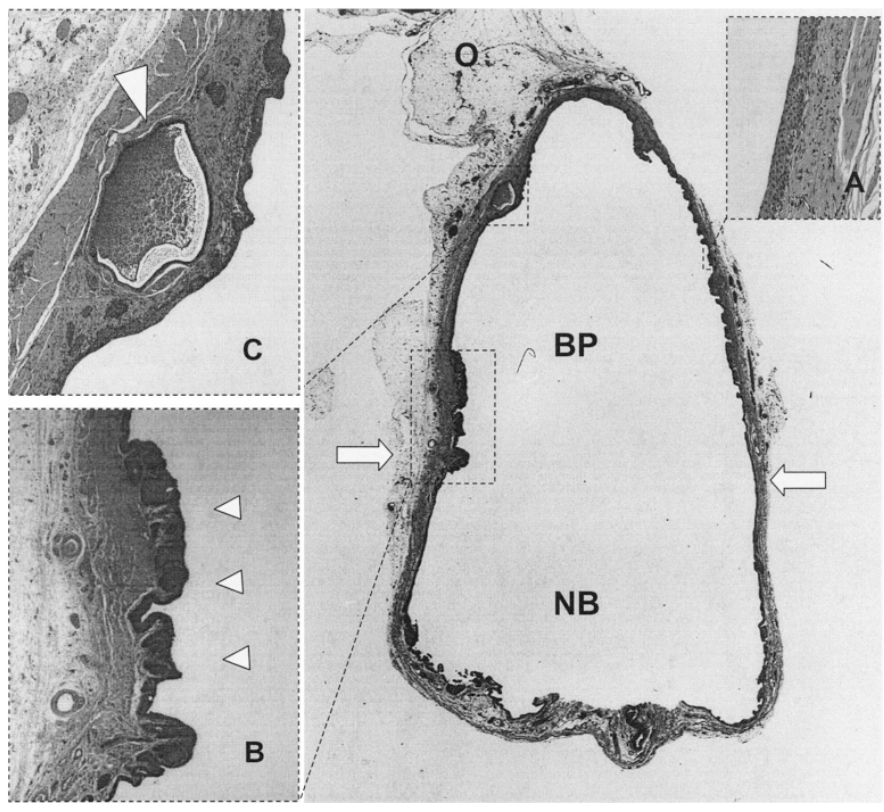

Figure 5. Bladder augmented by LPBTx (magnification: $\times 0.5$ ). Arrows indicate the vesicovesical anastomosis between the BP and the NB. O, omentum. The entire neobladder mucosa is normal (inset A) except for hyperplasia (arrowheads in the inset B) at the anastomosis, and a cyst (large arrowhead in inset C). Magnification in each inset: $\times 20$.

(9). This study was designed to be performed during a 2-y period, a time frame that corresponds with the life expectancy of a rat. We also chose 6-wk-old offspring as recipients to reflect the enhanced neovascularization and responsiveness of which younger tissue is capable. Essentially, the age of the bladder graft should match the age of the ileal patch as in previous experimental and clinical research because premalignant and malignant changes have usually developed near the anastomotic line, and we wanted to exclude as many etiologic factors as possible. Thus, any histopathologic changes around the anastomosis should be related to the anastomosis itself and not to differences in tissue age. The enhanced neovascularization that is capable of younger tissue is being exploited to facilitate the success of the partial transplantation process, whereas the older age of the ICP tissue is being used to assess for histopathologic transformations. This has direct relevance to later clinical application in humans because recipients will

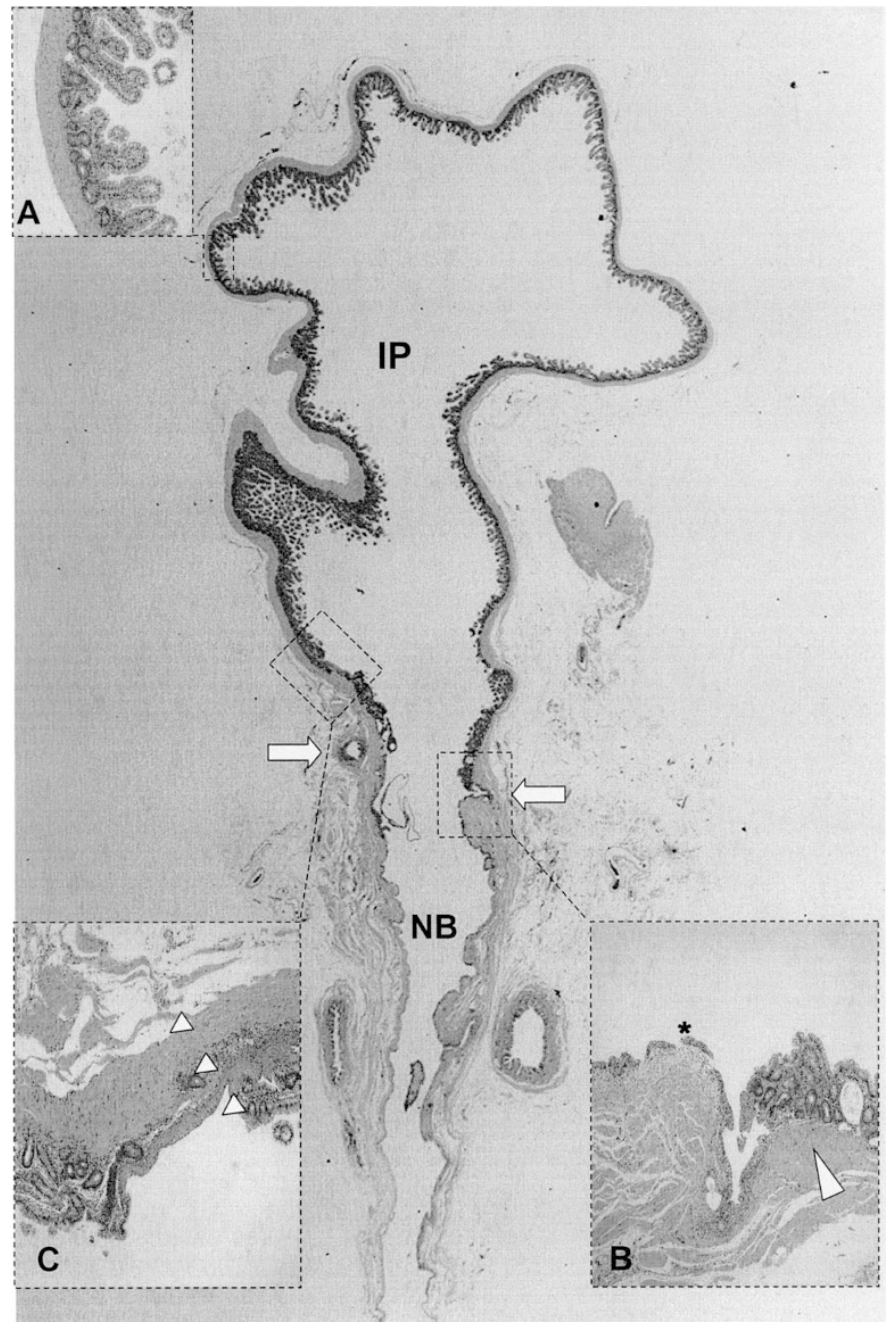

Figure 6. Bladder augmented by ileocystoplasty (magnification: $\times 0.5$ ). Arrows indicate the ileovesical anastomosis between the IP and the NB. Inset A shows normal IP mucosa, inset B shows hyperplasia (*) at the ileovesical anastomosis, and inset $C$ shows squamous metaplasia (arrowheads) in the IP mucosa near the anastomosis. The large arrowhead in inset B indicates suture material at the anastomosis site. Magnification in each inset: $\times 20$.

usually be children and donors would probably be older, but not necessarily living related.

The success of BA using LPBTx is partly because it is living-related transplantation and has a very short ischemic 


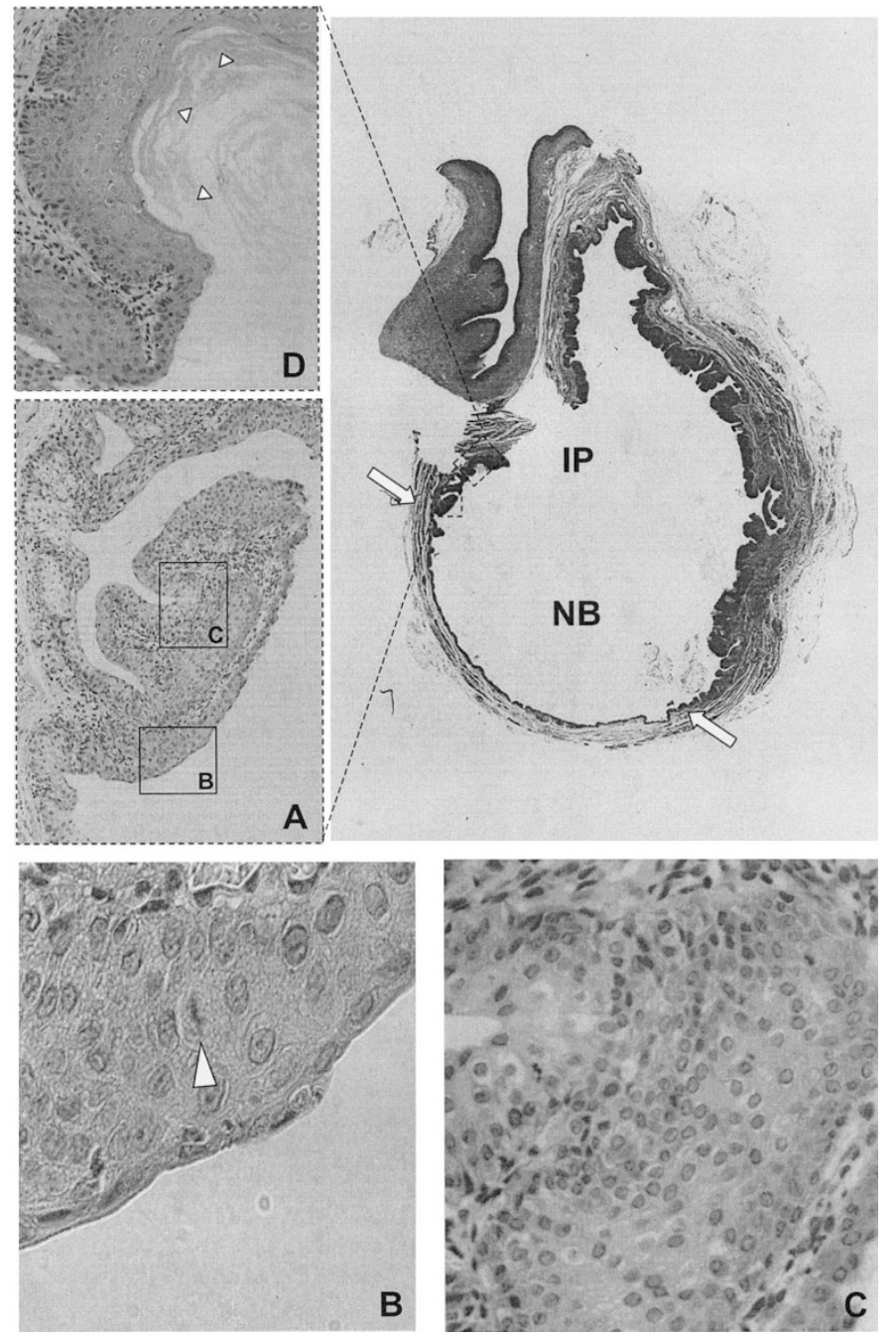

Figure 7. Bladder augmented by ileocystoplasty (magnification: $\times 0.5$ ). Arrows indicate the ileovesical anastomosis between the IP and the NB. Inset A shows dysplasia with mitosis in the IP mucosa near its anastomosis with the NB. High-power magnification clearly shows mitosis (inset B, large arrowhead) and dysplasia (inset C). Inset D shows squamous metaplasia (arrowheads) in the IP mucosa near the anastomosis. Magnification: $\times 10$ in inset A, $\times 100$ in inset $\mathrm{B}, \times 40$ in inset $\mathrm{C}$, and $\times 20$ in inset $\mathrm{D}$.

time compared with cadaveric transplantation and partly because in rats, the bladder wall is very thin and is easily neovascularized by the omentum. However, in larger animals with thicker bladders, such good results may not be possible because the thicker bladder wall may become necrotic because of ischemia. In fact, the bladder wall of a larger animal or a human may need to be trimmed by removing some layers to facilitate successful transplantation if our technique is in fact limited because of bladder wall thickness. This, however, remains to be assessed by further studies.

The MHC haplotype in Lewis rats is RT1 $\mathrm{A}^{1}(10)$. Because donors and recipients (offspring) both are Lewis rats, no rejection occurs, and no immunosuppressant was required in the present experimental model. However, should rejection need to be considered, for example, when this technique is tested in larger animals, it could strongly influence outcome. Nevertheless, immunosuppressant usage today is not fraught with the complications that hindered transplant success even up to a

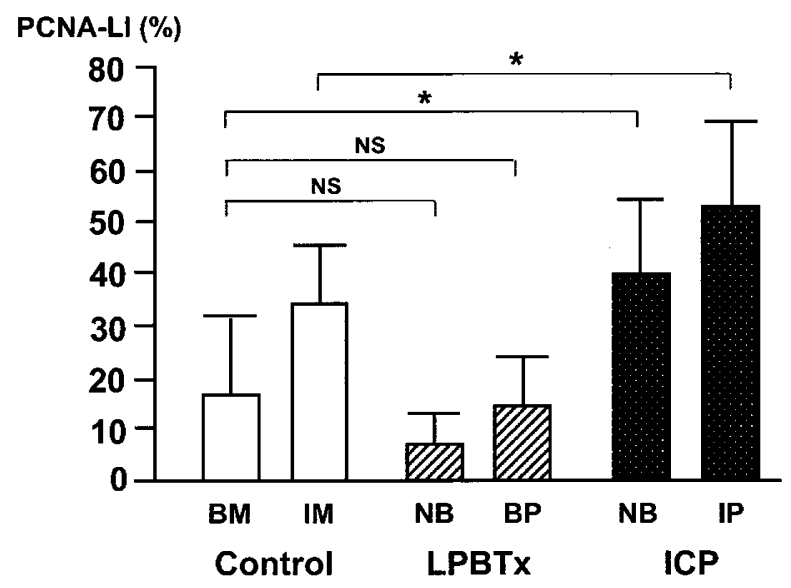

Figure 8. PCNA-LI in the LPBTx $(n=16)$ and ICP groups $(n=12)$. In ICP rats, PCNA-LI was significantly increased in both the NB and the IP mucosa near or at the vesico-intestinal anastomosis, compared with controls $(n=7$; both $p<0.05$ ). In contrast, there was no increase in PCNA-LI for both BP and NB in LPBTx rats. BM, bladder mucosa; IM, ileal mucosa. * $p<0.05$.

decade ago. We have used FK506 to prevent immunologic rejection without any side effects (11), and it causes complications at such a low rate in human liver transplantation that the complication rate is acceptable. LPBTx is applied for BA in humans; it will be necessary to perform LPBTx between rats with different MHC haplotypes and in larger animals as well to confirm that there are no detrimental sequelae on long-term follow-up.

In both LPBTx and ICP, occasional adhesive bowel obstruction is inevitable and caused complications in the present study. However, in LPBTx, urine leakage at the vesicovesical anastomosis is highly unlikely because the anastomotic line can easily be reinforced with omentum because the transplanted graft survives in the omentum, and we found that the omentum is larger and has more vessels for grafting. In contrast, in ICP, urine leakage at the vesico-intestinal anastomosis should be considered in the first instance if there are serious early postoperative complications. This was seen in the present study and has been reported clinically (12). Obviously, the omentum could theoretically be used to reinforce the anastomotic line in ICP cases as well, but the omentum is less vascular and less mobile in ICP cases because it has not been manipulated. Although theoretical, the omentum is not a reliable tissue, and also it is technically more difficult to use as a method for reinforcing the anastomosis in ICP cases. Thus, from a technical perspective, BA using LPBTx may be safer than ICP.

Alterations in $\mathrm{pH}$ and concentrations of sodium and potassium ions in urine have been reported to be important in stimulating proliferative changes (13), and Little et al. (14) reported that changes in serum $\mathrm{pH}$ and electrolyte concentrations secondary to augmentation cystoplasty can also play a role in the development of proliferative changes. However, previous studies did not evaluate these parameters or failed to note significant alterations in serum electrolytes or renal function in rats after augmentation cystoplasty $(14,15)$. We are the first to show that there are systemic changes in the ICP group, reflected as abnormalities in base excess, blood urea nitrogen, and serum $\mathrm{pH}$, biochemical 
complications that are being encountered clinically in children after augmentation cystoplasty $(1,16)$. In contrast, in LPBTx rats, there were no systemic biochemical changes identified probably because bladders were augmented with bladder mucosa rather than intestinal tissue.

The incidence of bladder calculi in rats with enterocystoplasty in other studies ranges from 14 to $33 \%(13,17,18)$ and ranges from 19 to $50 \%$ in human augmentation cystoplasty $(19,20)$, which is similar to the incidence (33.3\%) seen in this study.

The normal rat bladder has a capacity of $\sim 1 \mathrm{~mL}$ (9). BA increases bladder capacity, but as shown in this study, the neobladder can become abnormally large, especially in the ICP group. This phenomenon is also commonly seen after enteric cystoplasty in humans (20). Abnormal bladder enlargement contributes to urinary stasis and incomplete voiding. Enteric patches also produce mucus, and the high incidence of bladder calculi in enterocystoplasty is probably due to a combination of abnormally enlarged bladder, urinary stasis, and/or increased mucous production. In contrast, in BA using LPBTx, bladder capacities are increased but by less than double, and other factors are eliminated because the bladder is augmented with bladder tissue. Thus, there is likely to be a much lower incidence of bladder calculi in LPBTx compared with ICP (6.3 versus $33.3 \%$ ).

At the time of killing, ICP rats were significantly smaller than control or LPBTx rats. During follow-up, most rats in the ICP group seemed weaker and were killed before they actually died, to obtain adequate bladder specimens without autolysis. Thus, follow-up was shorter in the ICP group compared with the LPBTx group. Failure to thrive in ICP rats was probably due to metabolic disturbances, presence of bladder calculi, and/or urinary stasis as a result of abnormal bladder enlargement. Rats in both the LPBTx and the donor groups grew normally and were generally active during follow-up and at the time of killing. Strictly a control group having bowel anastomosis should have been included to determine the exact influence of bowel surgery on growth and general activity, and in the future, we will cover this to exclude the effect of bowel surgery alone. However, Sukhotnik et al. found that even though this study provided short-term results, the rats made a nice recovery and had normal growth after bowel resection, alone.

PCNA-LI increases in bowel with inflammation, dysplasia, or carcinoma and is a useful adjunct in the diagnosis of high-grade dysplasia $(6,7)$. High PCNA-LI in both the ileal patch mucosa and the native bladder mucosa may suggest that there is a higher potential risk for malignancy associated with ICP. In contrast, there was no significant increase in PCNA-LI in both the bladder patch and the native bladder in LPBTx.

Other investigators have reported carcinomatous changes in either the ileal patch mucosa or native bladder mucosa at or near the vesico-ileal anastomosis during long-term follow-up of rats with ICP $(14,16)$. Spencer et al. (15) reported an incidence of transitional cell carcinoma of $4 \%$ in ICP rats and $7 \%$ in colocystoplasty rats and an incidence of hyperplastic papillary lesions/transitional metaplasia of 54\% in ICP rats and $74 \%$ in colocystoplasty rats. In the present study, premalignant lesions such as dysplasia and mitosis were identified, with the incidence being $16.7 \%$ in the ileal patches in ICP, whereas there were no premalignant lesions seen in either the bladder patches or the native bladders in LPBTx despite that the follow-up period for the LPBTx group was longer than for the ICP group. Only chronic inflammation or hyperplasia was identified in LPBTx bladders, suggesting that bladders that are augmented using LPBTx are less likely to develop malignancy.

LPBTx seems to be associated with less post-BA complications such as metabolic acidosis, impaired linear growth, and bladder calculi than ICP. Furthermore, LPBTx may eliminate the risk for malignancy in the neobladder because bladder tissue is used for BA. The results of our long-term follow-up study suggest that LPBTx is associated with fewer complications when performed between rats of the same histocompatibility haplotype. In other words, there is strong justification for repeating this technique in rats of different histocompatibility haplotypes and larger animals with the aim of eventually using it in children who require BA. In conclusion, BA using LPBTx is safe and feasible as a technique, and further research into its potential for clinical application in humans is warranted.

\section{REFERENCES}

1. Gerharz EW, Turner WH, Kalble T, Woodhouse CRJ 2003 Metabolic and functional consequences of urinary reconstruction with bowel. BJU Int 91:143-149

2. Feng AH, Kaar A, Elder JS 2002 Influence of enterocystoplasty on linear growth in children with exstrophy. J Urol 167:2552-2555

3. Shokeir AA, Shamaa M, el-Mekresh MM, el-Baz M, Ghoneim MA 1995 Late malignancy in bowel segments exposed to urine without fecal stream. Urology 46:657-661

4. Filmer RB, Spencer JR 1990 Malignancies in bladder augmentations and intestinal conduits. J Urol 143:671-678

5. Yamataka A, Wang K, Okada Y, Kobayashi H, Lane GJ, Yanai T, Miyano T 2003 Living-related partial bladder transplantation for bladder augmentation in rats: an experimental study. J Pediatr Surg 38:913-915

6. Kullmann F, Fedate M, Gross F, Knuchel R, Bocker T, Steinbach P, Scholmerich J, Ruschoff J 1996 Expression of proliferating cell nuclear antigen (PCNA) and Ki-67 in dysplasia in inflammatory bowel disease. Eur J Gastroenterol Hepatol 8:371-379

7. Miyano T, Yamataka A, Iwashita K, Morioka A, Lane GJ, Kobayashi H, Okazaki T 2000 Histology of the neobladder mucosa after sigmoidocolocystoplasty. J Pediatr Surg 35:104-108

8. Atala A 2002 Future trends in bladder reconstructive surgery. Semin Pediatr Surg 11:134-142

9. Guan ZC, Ricard G, Charest-Boule L, Neilson K, Kiruluta G 1990 Augmentation cystoplasty in rats: development of an animal model. J Urol 144:461-465

10. Lee MD, Kunz HW, Gill TJ 3rd, Lloyd DA, Rowe MR 1986 Transplantation of the small bowel across $\mathrm{MHC}$ and non-MCH disparities in the rat. Transplantation 42:235-238

11. Wang K, Yamataka A, Kobayashi H, Hosoda Y, Miyahara K, Sueyoshi N, Lane GJ, Miyano T 2001 Transplantation of infantile bladder in rats: an alternative procedure for bladder augmentation. Transplantation 71:199-202

12. Nurse DE, Mundy AR 1993 Ileal augmentation cystoplasty. In: Webster G, Kirby R, King L, Goldwasser B (eds) Reconstructive Urology, Vol 1. Blackwell Scientific Publications, Boston, pp 421-431

13. Lina BA, Woutersen RA 1989 Effects of urinary potassium and sodium ion concentrations and $\mathrm{pH}$ on N-butyl-N-(4-hydroxybutyl) nitrosamine-induced urinary bladder carcinogenesis in rats. Carcinogenesis 10:1733-1736

14. Little JS Jr, Klee LW, Hoover DM, Rink RC 1994 Long-term histopathological changes observed in rats subjected to augmentation cystoplasty. J Urol 152:720-724

15. Klee LW, Hoover DM, Mitchell ME, Rink RC 1990 Long-term effects of gastrocystoplasty in rats. J Urol 144:1283-1287

16. Spencer JR, Steckel J, May M, Marion D, Hernandez K, Vaughan ED Jr 1993 Histological and bacteriological findings in long-term ileocystoplasty and colocystoplasty in the rat. J Urol 150: 1321-1325

17. Buson H, Diaz DC, Manivel JC, Jessurun J, Dayanc M, Gonzalez R 1993 The development of tumors in experimental gastroenterocystoplasty. J Urol 150:730-733

18. Wang K, Yamataka A, Morioka A, Lane GJ, Iwashita K, Miyano T 1999 Complications after sigmoidocolocystoplasty: review of 100 cases at one institution. J Pediatr Surg 34:1672-1677

19. Vajda P, Kaiser L, Magyarlaki T, Farkas A, Vastyan AM, Pinter AB 2002 Histological findings after colocystoplasty and gastrocystoplasty. J Urol 168:698-701

20. Miyano G, Yamataka A, Okada Y, Shimotakahara A, Kaneko K, Lane G J, Yamashiro Y, Miyano T Sigmoidocolocystoplasty for augmentation of iatrogenic small capacity bladder caused by direct injury to the bladder during inguinal hernia repair: long-term follow-up. Pediatr Surg Int 20:61-64 